\title{
Intelligent Healthcare Systems Assisted by Data Analytics and Mobile Computing
}

\author{
Xiao Ma, ${ }^{1}$ Zie Wang, ${ }^{1}$ Sheng Zhou, ${ }^{1}$ Haoyu Wen, ${ }^{1}$ and Yin Zhang $\mathbb{D}^{1,2}$ \\ ${ }^{1}$ School of Information and Safety Engineering, Zhongnan University of Economics and Law, Wuhan, China \\ ${ }^{2}$ State Key Laboratory for Novel Software Technology, Nanjing University, China \\ Correspondence should be addressed to Yin Zhang; yinzhang@zuel.edu.cn
}

Received 9 January 2018; Accepted 20 May 2018; Published 3 July 2018

Academic Editor: Javier Prieto

Copyright (c) 2018 Xiao Ma et al. This is an open access article distributed under the Creative Commons Attribution License, which permits unrestricted use, distribution, and reproduction in any medium, provided the original work is properly cited.

\begin{abstract}
It is entering an era of big data, which facilitated great improvement in various sectors. Particularly, assisted by wireless communications and mobile computing, mobile devices have emerged with a great potential to renovate the healthcare industry. Although the advanced techniques will make it possible to understand what is happening in our body more deeply, it is extremely difficult to handle and process the big health data anytime and anywhere. Therefore, data analytics and mobile computing are significant for the healthcare systems to meet many technical challenges and problems that need to be addressed to realize this potential. Furthermore, the advanced healthcare systems have to be upgraded with new capabilities such as machine learning, data analytics, and cognitive power for providing human with more intelligent and professional healthcare services. To explore recent advances and disseminate state-of-the-art techniques related to data analytics and mobile computing on designing, building, and deploying novel technologies, to enable intelligent healthcare services and applications, this paper presents the detailed design for developing intelligent healthcare systems assisted by data analytics and mobile computing. Moreover, some representative intelligent healthcare applications are discussed to show that data analytics and mobile computing are available to enhance the performance of the healthcare services.
\end{abstract}

\section{Introduction}

In the past two decades, advanced information technologies, such as mobile communication systems [1], big data [2], Internet of Things (IoT) [3], and wearable computing [4], have been widely used in the sector of healthcare [5]. Particularly, various novel healthcare systems assisted by big data and mobile computing are developed for provide intelligent and professional services [6]. However, the explosion of healthcare data brings the following challenges for data management, storage, and processing:

(i) Large Scale. With the improvement of electromedical and wearable devices, the data volume of healthcare systems has been extensively increasing [7].

(ii) High Throughout. Generally, major electromedical and wearable devices can continuously acquit health data, while these data need to be processed rapidly for prompt response to emergencies [8]. (iii) Various Forms. There are various healthcare data generated and stored in healthcare systems, such as medical record, hospitalization records, medical imaging, and surgery data. These multisourced data include text, image, audio, and video [9]. More importantly, the same category of healthcare data collected through different devices may follow the different data standard defined by providers.

(iv) Deep Value. The value of mining single source healthcare data is very limited. Thus, more research attempted to develop data fusion based approach to discover more knowledge from various data to provide more valuable services, such as personalized health guidance and public health warnings [10].

Fortunately, with the assistance of advanced techniques, more intelligent healthcare services are supported by data analytics, while it becomes more convenient for users to access to these novel services [11]. For example, M. Pramanik 
et al. propose a big data enabled smart healthcare system framework to offer theoretical representations of an intraand interorganizational business model in the healthcare context [12]. M. Rathore et al. developed a Hadoop-based intelligent healthcare system demonstrating IoT-based collaborative contextual big data sharing among all of the devices in a healthcare system [13]. S. Peddia et al. designed a cloudbased mobile e-health calorie system that can classify food objects in the plate and further compute the overall calorie of each food object with high accuracy [14].

Although the great innovation is happening in the healthcare field, there are several issues need to be addressed, especially the heterogeneous data fusion, mobile data transmission and analysis, etc. [15-17]. In [18], it discussed clear motivations and advantages of multisensor data fusion and particularly focuses on physical activity recognition, aiming at providing a systematic categorization and common comparison framework of the literature, by identifying distinctive properties and parameters affecting data fusion design choices at different levels (data, feature, and decision). In [19], it presented the electronic health record big data analytics for precision medicine, including data preprocessing, mining, and modeling.

Nowadays, a huge number of researches focus on data analysis or data mining for healthcare data $[10,20]$ on technical details in deploying and implementing mobile computing $[21,22]$, but one of the greatest challenges is how to develop a comprehensive healthcare system for effectively manage multisource heterogeneous healthcare data with particular technical features. Thus, this paper presents a detailed design of intelligent healthcare systems assisted by data analytics and mobile computing, and it make the following contributions: (1) It proposes a unified data collection layer for integrating the healthcare data from public sources and personal devices. (2) It establishes a cloud-enabled and data-driven platform for multisource heterogeneous healthcare data storage and analysis. (3) It designs a healthcare application service layer to provide unified application programming interface (API) for developers and unified interface for users.

\section{Related Technologies}

2.1. Mobile Computing. Mobile computing is an emerging technology related to multiple disciplines and is involved in many areas; it is also a hot issue in computer technology research. Mobile computing concerns how to provide quality information services (information storage, query, calculation, etc.) to mobile users (including users on laptops, mobile phones, and pagers) distributed across different locations. Mobile computing is a new type of technology that enables computers and other information devices to transmit data without being connected to a fixed physically connected device [23]. With the increase in mobile device usage, mobile computing is booming and has begun to be applied to different fields. Related work has been done in the education field [24].

In the medical field, mobile computing not only plays an important role but also is a very meaningful application direction as a supporting technology for mobile healthcare.
Medical professionals are using mobile devices to change clinical practice. Currently, many medical software applications can help people perform nursing tasks, from information and time management to clinical decision making [25].

The use of mobile devices, healthcare professionals (HCPS), by healthcare workers has changed many aspects of clinical practice, and it has become commonplace in healthcare environments, leading to the rapid development of medical applications. Many applications can now assist healthcare professionals in many important tasks, including information and time management, health record maintenance and access, communication and counseling, information reference and gathering, patient management and monitoring, clinical decision making, medical education, and training. Current mobile devices and applications provide many benefits to HCPs, where the most significant benefit is that people can be better cared for since they support clinical decision making and help patients recover. In [25], the following benefits of mobile devices in healthcare are summarized: convenience, better clinical decision making, and improved accuracy. Mobile computing can not only improve the accuracy of identifying relevant information and the accuracy of metrics but also increase efficiency and productivity.

An effective mobile computing platform must be able to effectively use semantic-rich and medically plausible inferences about physiology, physiology, and psychology by sensing sensor information and behavioral status and linking these inferences with environmental, social, and other factors [26]. The challenge facing mobile computing is the issue of energy consumption, especially in the medical field, which often requires long monitoring durations and lengthy testing of patient-related physiological indicators. In [27], Kao et al. studied the energy consumption and performance of mobile computing, therein developing a task assignment problem and providing a dynamic programming algorithm. Hermes is a solution to the optimal strategy problem for balancing the issues of improving latency and energy consumption of mobile devices.

With the increasingly in-depth research being performed, mobile computing will have a better future in the medical field.

2.2. Big Data. Huge amounts of data are generated in the medical field, therein increasing rapidly every day, especially in mobile healthcare. Normal mobile devices and wearable devices usually need a long time to detect the related physiological indices of the human body, but mobile medicine makes obtaining a patient's physiological data more convenient and accurate, and it has greatly facilitated the development of medical big data.

Big data also provide additional data support for medical treatment, such as in medical imaging and processing, electronic health records, epidemiology, and other higher level analyses of healthcare data and can play an auxiliary role in medical diagnosis [28]. In [29], Viceconti et al. proposed that big data analysis can be successfully combined with VPH technologies to create new electronic medical solutions that are both effective and robust. 
In mobile healthcare, mobile computing technologies can utilize big data technologies to perform relevant analysis and processing to obtain related data in a timely and convenient manner to provide better medical care. Lv et al. [30] introduced two mobile healthcare applications that can serve as health services based on big data. On the one hand, big data can play a role in electronic medical record collection terminals; on the other hand, big data provide doctors with solutions for developing rehabilitation tools.

In [31], the effects and benefits of big data analytics for healthcare were illustrated, therein suggesting that big data analytics may change the way healthcare professionals gain insights from their clinical and other data repositories and make informed decisions using cutting-edge technology.

However, big data technologies also face many challenges in the healthcare industry. Belle et al. [32] discussed three important up-and-coming and important areas of medical research: image-, signal-, and genomic-based analysis. In the medical field, the use of a large amount of medical data and related analytical techniques can provide a positive impact.

2.3. Cloud Computing. Cloud computing is the addition, use, and delivery of Internet-based services and often provides dynamically scalable and virtualized resources through the Internet [33]. The development of cloud computing is not limited to PCs; with the vigorous development of the mobile Internet and the emergence of various mobile terminal devices, mobile cloud computing services have emerged. Cloud computing and big data technology are inseparable and are often used in combination [34]. In [35], a networked physical system based on patient-centered healthcare applications and services was proposed and called Health CPS. It builds on cloud and big data analytics. The results of their research show that cloud and big data technologies can be used to improve the performance of medical systems and allow people to enjoy a variety of smart medical applications and services.

By combining the advantages of these two technologies, mobile cloud computing can be better applied to mobile healthcare. If certain limitations, such as limited memory, CPU power, and battery life, can be overcome, mobile cloud computing could greatly improve the capabilities and effects of mobile and cloud computing.

Loai A. et al. [36] proposed the design of networked healthcare systems using big data and mobile cloud computing technologies. Wan et al. [37] studied a cloud-enabled WBAN architecture and its applications in pervasive healthcare systems. Using energy-efficient routing, cloud resource allocation, semantic interaction, and data security mechanisms, the system transmits critical sign data to the cloud, and it provides tremendous opportunities for healthcare systems.

Certain benefits brought by cloud computing, such as the scalability offered by "potential" users (i.e., pay-as-yougo users) and software and virtual hardware services being delivered online (for example, collaborative planning, virtual servers, and virtual storage devices), will ensure that organizations do not have to maintain and update their software and hardware facilities by themselves. The flexibility of this emerging computing service can create many possibilities for organizations that currently do not exist [38].

2.4. Wearable Computing. In recent years, various mobile devices have emerged, including healthcare and medical devices (such as sports wristbands, watches, and smartphones). These wearable devices have computing abilities to record or detect relevant data for the user. Wearable computing technology has greatly facilitated mobile healthcare, as many wearable devices with disease monitoring and body perception abilities have been proposed [39, 40].

The development of wearable computing involves more aspects, and Chen et al. [41] proposed a new architecture for wearable computing based on emotional interaction and cloud technology design mechanisms; they then explored its current problems to demonstrate potential research in new directions. With the development of wearable devices, the wearable computing power needs to be further improved to better assist in medical and healthcare. Relevant studies on, e.g., computing power, connection methods, and power consumption, have been conducted [42-44]. Mobile cloud computing, big data, and other technologies are also promoting the development of wearable computing toward providing better technical support to mobile healthcare.

2.5. Internet of Things. Both wearable devices and mobile terminal devices are inseparable from the Internet of Things. The Internet of Things technology is one of the key technologies for these devices for transmitting and retrieving data and information. Its smart sensing technology provides important support for mobile medical data transmission and acquisition [45]. Catarinucci et al. [46] proposed a smart hospital system (SHS) that relies on different but complementary technologies, particularly wireless sensor networks, RFID, smartphones, and interoperability. SHS can collect real-time environmental conditions and patient physiological parameters.

Internet of Things technology, big data technology, and cloud computing are popular and widely used technologies and are often interdependent. Advanced terminal technologies (e.g., smart apparel) and advanced cloud computing technologies (e.g., big data analytics and cloud cognitive computing) are expected to provide people with more reliable and intelligent services.

In [4], Chen et al. presented a wearable medical 2.0 system to enhance QoE and QoS for the next generation of healthcare systems. In this system, washable smart clothing, including sensors, electrodes, and leads, is the key component of cloud analytics that provide users with their physiological data and receive the health and emotional state of machinebased, intelligent users. In emergency services, IoT can collect, integrate, and interoperate IoT data to flexibly support emergency medical services. The results of [47] show that the resource-based IoT data access method is effective in distributed and heterogeneous data environments and can access data across cloud and mobile computing platforms in a timely manner.

Internet of Things technology is also commonly used at home and is often connected with home healthcare [48]. The 
seamless convergence of IoT devices in various platforms (such as sensors and wearable smart medicine kits) improves the user experience and service efficiency of home healthcare services (such as telemedicine).

2.6. Cyber Physical Systems. As a unity of computing processes and physical processes, cyber physical systems (CPSs) represent the next generation of intelligent systems, therein integrating computing, communications, and control [49]. The information physical system interacts with physical processes through a human-computer interaction interface and enables the networked space to manipulate a physical entity in a remote, reliable, real-time, secure, and collaborative manner.

The information physical system includes future ubiquitous environmental awareness, embedded computing, network communication, network control, and other types of system engineering; it also provides the functions of computing, communication, precise control, remote collaboration, and autonomy. In addition, such a system focuses on the close combination and coordination of computing resources and physical resources. It is mainly used in certain intelligent systems such as equipment interconnection, IoT sensors, smart homes, robotics, and intelligent navigation systems.

As a prominent subcategory of networked physical systems, to generate a convenient and cost-effective platform and promote complex and ubiquitous mobile sensing applications between humans and the surrounding physical world, mobile devices, such as smartphones, are widely used in mobile networked physical systems [50].

In [51], Costanzo et al. presented a flexible and reliable monitoring system based on embedded systems and wearable devices that allows doctors and family members to monitor the patient's distance using a cell phone. In emergency situations, proper communication with the emergency center is required to rescue patients promptly. Thus, the system can effectively monitor the health of elderly individuals.

With the rapid development of IT systems, embedded software has replaced the monitoring and diagnosis of patients. However, these systems have limitations in a wide range of device interoperability and data aggregation aspects. To solve these problems, medical network physical systems (MCP systems) have gradually become a new paradigm for medical systems.

The paradigm introduced by mobile networked physical systems (MCPs) and Time Interventions (TI) transcends traditional medical environments in terms of the monitoring, diagnosing, treating, and management of patient health. These paradigms can provide medical care to patients at any place and at any time. Among them, MCP provides the necessary technical support system that facilitates collective work in an autonomous manner.

\section{Intelligent Healthcare System Architecture}

3.1. Design Issues. With the increasing cost of healthcare services and medical insurance, people need more aggressive health and wellness monitoring. In the medical industry, big data and cloud computing are gradually becoming trends

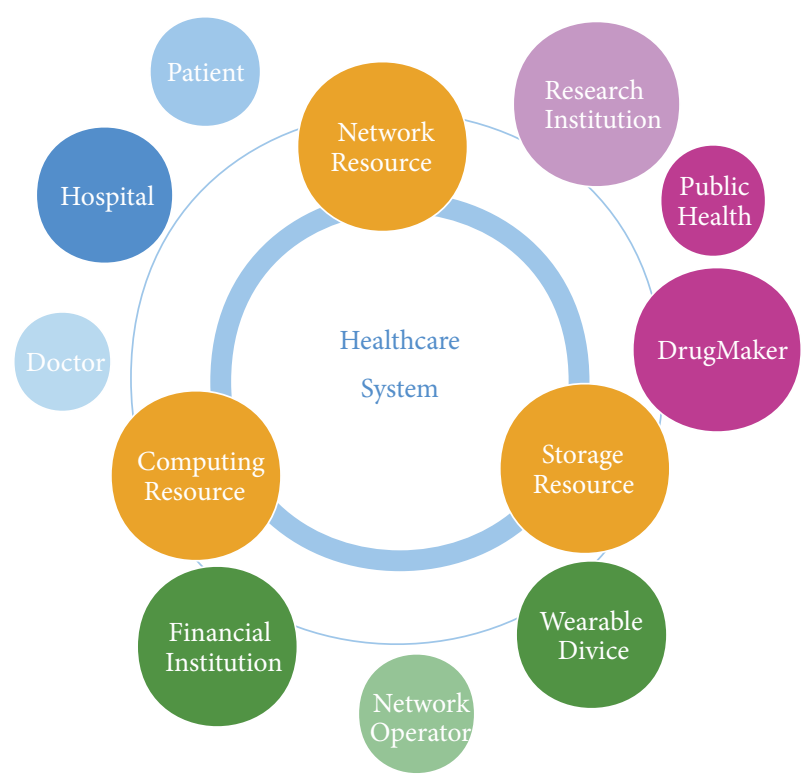

FIGURE 1: Expanded healthcare system.

for medical innovation. As a result, the medical industry is experiencing an increase in the amount of data generated in terms of complexity, diversity, and timeliness; the industry increasingly relies on the collection and analysis of data. Therefore, to make better decisions, we need to collect data and conduct effective analysis. The cloud is a good choice for on-demand services for storing, processing, and analyzing data. Medical data released and shared through the cloud are very popular in practice, and information and knowledge bases can be enriched and shared through the cloud.

The revolution presented by the cloud and big data can have a huge impact on the healthcare industry, and a new healthcare system is evolving. Figure 1 shows an expanded healthcare system that includes traditional roles (such as patients and healthcare providers) and other new members. This is why we need to design a more appropriate healthcare system to meet the challenges presented by this revolution.

(i) The diversity of data sources requires a uniform standard of heterogeneous data management. On the one hand, due to the diversification of medical equipment, the data formats and the amount of data generated by various devices may be quite different, which requires that the system support data access by various medical devices to ensure high scalability and satisfy actual medical needs. On the other hand, the system needs to convert the received data into a unified standard to improve the efficiency of data storage, query, retrieval, processing, and analysis.

(ii) The diversity of data content requires a unified programming interface for multiple data analysis modules. Analytical techniques have also been extended to require complex analysis for accommodating the four characteristics (4Vs) of big data in the medical field due to inconsistencies in the sources, structures, functional scenarios, and nature of health 


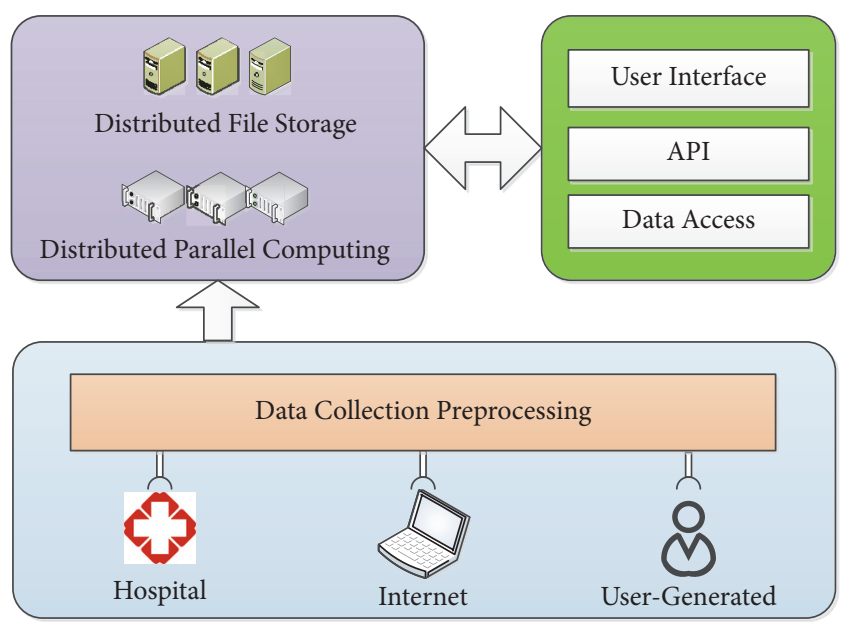

FIGURE 2: Architecture of intelligent healthcare systems assisted by data analytics and mobile computing.

data: volume, velocity, veracity, and variety [31]. Gone are the days of collecting data on electronic health records and other structured formats. Diversified medical data, including structured, semistructured, and other unstructured data, represent one aspect making medical data both interesting and challenging. Based on different data structures, the system can efficiently deploy and analyze data online or offline, such as via stream processing, batch processing, iterative processing, and interactive query, therein reducing system complexity and improving development and access efficiency.

(iii) The diversity of service objects requires a uniform standard service platform interface. Medical data that have previously been processed also have different data contents, data formats, data amounts, etc. with respect to different service targets; that is, the system can provide different applications and services with respect to the different roles of the service object. To provide reliable medical services, resource optimization, technical support, and data sharing are crucial to a system's application service platform.

3.2. Cloud- and Big-Data-Assisted Architecture. Figure 2 shows the architecture of intelligent healthcare systems assisted by data analytics and mobile computing, including the data acquisition layer, data management, and application service layer.

(i) Data Acquisition Layer. The main function of this layer is to collect user medical data and provide standardized data for a unified standard of multivariate data management through adapter preprocessing. This section consists of a variety of data nodes and adapters, primarily from hospitals (hospital information systems and electronic medical records), Internet (social networking services and real-time communication), and user-generated content (terminal equipment and wearable equipment). Adapters can preprocess and encrypt raw data from various devices to ensure the security and availability of data transmitted at the data management layer.

(ii) Data Management Layer. To support the efficient management and analysis of medical data, this layer consists of a Distributed File Storage (DFS) and Distributed Parallel Computing (DPC). DFS provides highly efficient data storage, high-throughput data upload and download, fast data retrieval. and exchange capabilities for heterogeneous medical data to improve system performance; the DPC module analyzes and processes data from the DFC module for big data. The scale of unstructured data provides offline calculations and provides the appropriate processing and analysis methods based on the timeliness of the data and the priorities of the tasks.

(iii) Application Service Layer. This layer consists of three parts, the user interface, API, and data access, which provides users with basic visual data analysis results. Through the data access module, following the data management layer analysis of the data, application developers can use API scheduling through the user interface to provide rich, professional, and personalized medical services.

\section{Data Collection Layer}

At one end, mHealth is a physician, and at the other end, it is a user with demands. The middle consists of a service provider that bridges the two ends with a variety of technologies and tools, including mobile network operators, mobile network technologies and equipment providers, mobile terminal manufacturers, IT companies (including software and hardware suppliers and system integrators), financial investors, insurance companies, public health medical institutions, banks and payment companies, private healthcare institutions, pharmaceutical companies, healthcare providers, research centers, government and nongovernmental organizations, and solution providers and more.

Data have become a particularly important aspect of mobile health. Data collection requires both the collection of devices (cell phones, computers, and portable devices) and software for gathering information. The data mainly concern the visualization of static text but can also be extended to interactive decision support algorithms, other visual image information, and communication via email and SMS integration. The consolidation of the use of geomapping components using GIS and GPS Mobile technologies is used to "tag" voice and data traffic to specific locations or to a range of locations. These combined capabilities have been used for emergency health services as well as disease surveillance, the mapping of health facilities and services, and other healthrelated data collection processes.

Usually, medical data classification can be divided into two aspects. One aspect is the class of data for the hospital in the operation, and the operation will produce a series of data. The other aspect is "clinical" data, which is unique to the healthcare industry. The same classification can be performed 


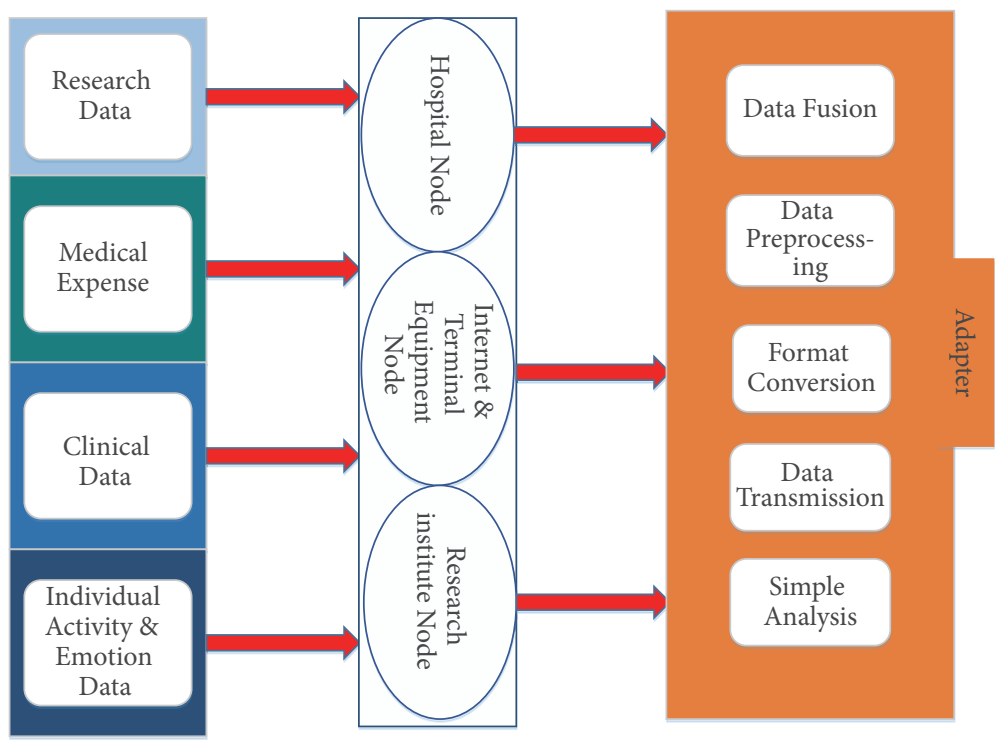

FIgURE 3: Data collection layer.

for personal, institutional, government, and healthcare information. The development of mobile healthcare has gradually transformed the main providers of medical data from hospitals to intermediaries or the government to make the data as public as possible without infringing upon the privacy of others. As shown in Figure 3, the data collection layer consists of data nodes and adapters.

4.1. Data Nodes. Based on mobile medical device conditions, a user-friendly intelligent healthcare system (mobile health) collects not only traditional medical data but also medicalrelated daily behavioral data. According to the different types of data, data nodes can be divided into the following four categories:

(i) Research organization nodes. This node mainly concerns the research and development of medical equipment companies, R \& D outsourcing companies, research institutions, and other data generated during the development of the main sources of data, including (1) medical research and development processes, such as hospital clinical trials, and (2) the latest scientific research institutes. Drug R \& D institutions and life science research institutions, such as major medical colleges and universities and major drug research and development centers, have accumulated a wealth of research data such as clinical trial data and high-throughput screening data. These numerical data, including personal and clinical gene and protein data, can help identify drug side effects and new effects.

(ii) Hospital nodes. In this node of the hospital, one can include most medical data, such as clinical data and medical expenses. Clinical data are a type of medical data usually collected by medical service providers for the clinical diagnosis and medical imaging of
EMR, etc. These data can be unified, managed, and made open to researchers to ensure the necessary prerequisites of patient privacy and to maximize the clinical data dig value. For data generated during a hospital clinic visit, the main collection port is a medical institution such as a hospital. These data include electronic medical records, traditional test results (biochemistry, immunology, PCR, etc.), emerging test results (gene sequencing, etc.), doctors' prescriptions, and pathways for diagnosis and treatment. These data include rapidly growing segments, especially emerging test data, such as genetic test data. In addition, in this node, the medical behavior produces important cost data. The cost data refer to all audit/reimbursement records related to the payer, including patient payment records, reimbursement records, and medical circulation records such as medical expenses and medical treatment insurance claims. These data are not traditional medical data but can be used to analyze and estimate medical costs. These data are usually stored in different geographic locations in the medical institution's database using a unified data format.

(iii) Internet and endpoint devices. Personal activity and emotion as well as patient health metric data are generated at this node. These data generally refer to the patient's own, out-of-hospital behavior, and sensory data, and the main collection terminals are wearable devices and various online light medical platforms, including (1) the health management of signs collected through wearable devices data and (2) network behavior data such as registered medical consultations, online pharmacies, health management, and patient-patient exchanges. This aspect of the data can also be generated from third-party 
mobile device providers (the Internet) based on mobile medical devices. These data can play a significant role in personal healthcare recommendations. Simple analysis includes personal retail consumption records reflecting lifestyle habits that can be used to assess personal health risks and develop personalized health plans. Based on the physiological data collected by wearable devices, the user's health can be easily monitored and tracked. Personal emotion data can be collected through information posted on social networks and can be used for mental health measures and emotion calculations. Particularly for the rehabilitation of patients, doctors can adjust their treatment plan based on the patient's emotions. The emotional perception of medical services with human treatments can be used to promote innovations in modern medicine [52].

4.2. Adapter. Adapter is a data node that provides access to system middleware, not simply the physical data link or the original data preprocessor and encryptor. In addition to cleaning up the data, removing redundancy, and compression, the preprocessing module also supports data format conversion. Depending on the type of data collected, the adapter uses a system-defined data standard for format conversion. The encryption module encrypts the preprocessed data to ensure security via hierarchical privacy protection. Unauthorized devices cannot decrypt packets even if they have access to the system. To improve the scalability of this system, the functional unit of the adapter is configurable. When the following basic conditions are met, the corresponding module of the adapter can be updated online.

(i) Data Node Changes. When a data node is replaced or upgraded, the functional unit will not work properly if the data format of the updated device is different from the previous version. The adapter must then send a request to the server to reconfigure the preprocessing module to be compatible with the updated module, and the server records the type of the updated data node and reauthorizes the encryption module online.

(ii) Data Standard Updates. When a new type of device without a system-defined data standard can access the system, the data standard library should be extended and is expected to be pushed to the appropriate module for updating [35].

In general, the adapter needs to implement the following five modules:

(i) Data Fusion. From the perception layer to the application layer of the Internet of Things, the types and quantities of various information are exponentially increasing. The amount of data that needs to be analyzed also increases in stages. This involves various heterogeneous networks or systems. Data fusion fuses multisensor information source data and information to obtain more accurate position estimations and identity estimations, being combined with P2P, cloud computing, and other distributed computing technologies. The above-mentioned data nodes, research data, clinical data, medical expenses, personal activity, and emotion data are effectively used to integrate, mine, and intelligently process vast amounts of data.

(ii) Preprocessing. Preprocessing refers to the preprocessing of data before the main processing. For example, before converting or enhancing most medical clinical data, the first step is to convert the irregularly distributed data into a regular distribution to facilitate operations by a computer.

(iii) Format Conversion. The data in the system change frequently. If there is no format conversion during data node replacement or upgrade, the data format will be inconsistent with the previous version; the function unit will then no longer work properly.

(iv) Data Transmission. The system is designed for user data transmission, which can be performed via $\mathrm{WiFi}$, $4 \mathrm{G}$, Bluetooth, and other wireless networks; for example, a user-facing system eliminates the need for patients to visit hospitals for live health data when they provide patients with personalized and intelligent monitoring of health data in real time. This can be based on cloud computing to provide intelligent health services, therein integrating a variety of wireless health monitoring equipment such as blood pressure monitors, peak flow meters, glucose monitors, scales, and ECG monitors. These devices are used by patients who need remote monitoring. The readings received from such devices are automatically forwarded to preconfigured mobile devices (mobile phones, PDAs, laptops, etc.) via Bluetooth, $\mathrm{WiFi}$, and $4 \mathrm{G}$ cellular mobile networks. As a result, the patient's health data can be securely and accurately transmitted to a cloud-based web service without requiring the patient to have IT expertise and without user intervention [53].

(v) Simple Analysis. A simple analysis consists of the data collected from end devices, wearable devices, and the above-mentioned data nodes as well as a summary of the results. For example, some data sent by a social network, such as heartbeat condition, sleep quality, diet, fat, and calorie consumption, can be monitored by a wearable device (iWatch) to reflect the user's living conditions for implementing intelligent real-time healthcare system monitoring functionality [54].

\section{Data Management Layer}

As shown in Figure 4, this layer consists of a Distributed File Storage (DFS) module and a Distributed Parallel Computing (DPC) module. Using big-data-related technologies, DFS will improve system performance by providing efficient data storage and I/O for heterogeneous medical data. Based on the timeliness of the medical data and the priority of the task, DPC provides the appropriate treatment and analysis. 


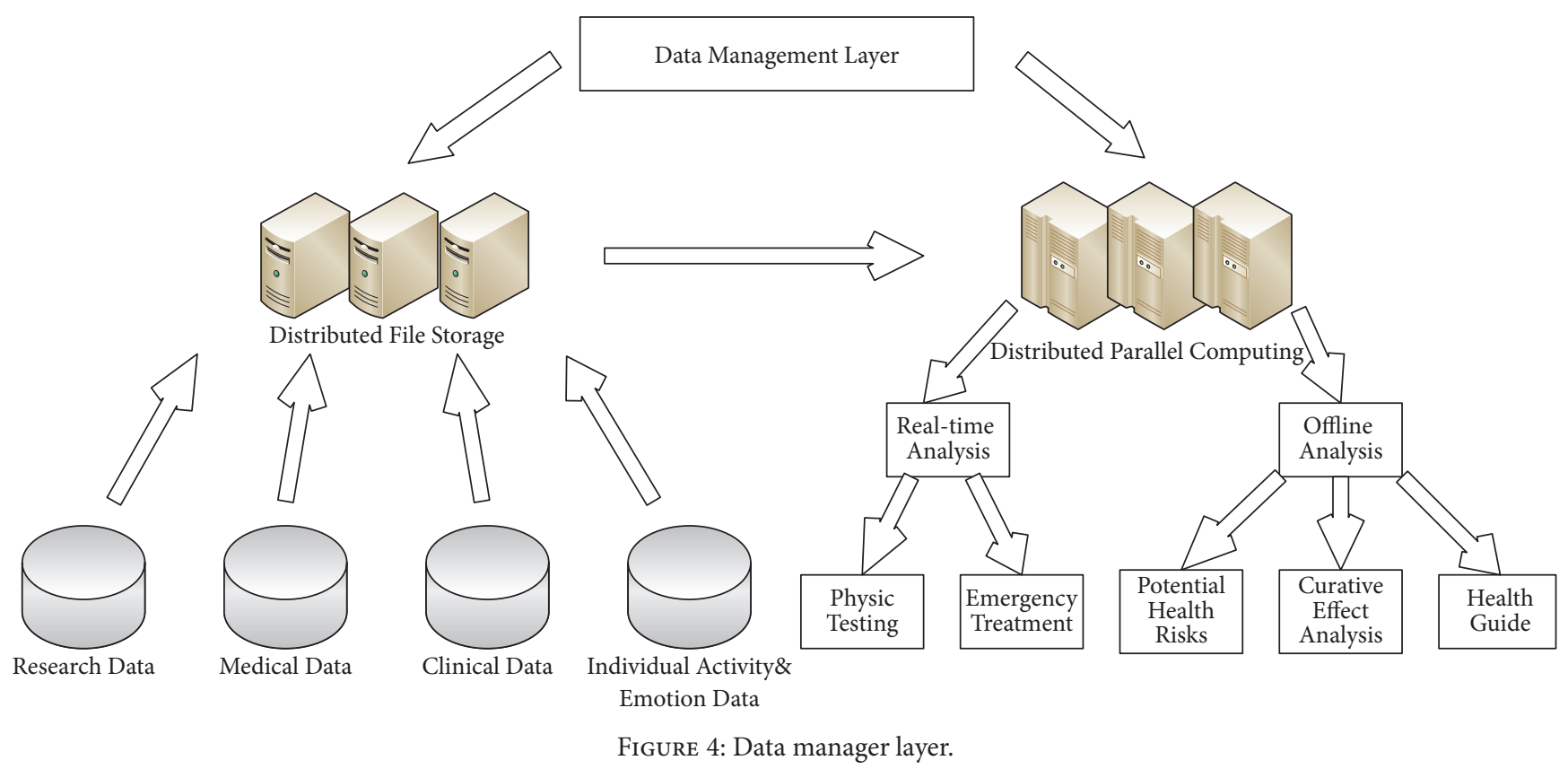

5.1. Distributed File Storage Module. Distributed storage systems use multiple servers to synergistically store data. Traditional network storage systems use a centralized storage server to store all the data. The storage server becomes the bottleneck for system performance and is the focus of reliability and security; traditional servers cannot meet the needs of large-scale storage applications. Distributed network storage systems adopt a scalable system architecture that utilizes multiple storage servers to share the storage load and uses the location server to locate and store information. This not only improves system reliability, availability, and access efficiency but also is easily expandable.

The distributed storage architecture consists of three parts: the client, the metadata server, and the data server. The client is responsible for sending read and write requests, cache file metadata, and file data. The metadata server is responsible for managing the metadata and processing client requests and is the core component of the entire system. The data server is responsible for storing the file data to ensure the availability and integrity and the data. The benefits of this architecture are that both performance and capacity can be expanded simultaneously, and the system is highly scalable.

Distributed storage is facing more complicated data needs, which can be divided into three categories.

Unstructured data: unstructured data include all formats of office documents, text, images, audio, and video information.

Structured data: structured data are stored in data relational libraries; one can use two-dimensional relational table structure representations. The structured data schema (schema, including attributes, data types, and the links among data) and the content is separate, and the data model needs to be predefined.

Semistructured data: between unstructured data and structured data, HTML documents belong to the semistructured data category. Such data are generally selfdescribing, and the biggest difference from structured data is that the schema structure and content of semistructured data are mixed, with no obvious distinction and no schema structure that predefines the data.

The main challenge facing large-scale healthcare data is how to create an efficient, mass data distributed storage mechanism and how to support efficient data processing and analysis.

For large-scale medical data, traditional relational databases obviously cannot meet big data challenges. NoSQL database [55] has a flexible model that supports easy-to-use replication, simple APIs, eventual consistency, and support for large amounts of data. This section describes the three main NoSQL databases, each based on a specific data model.

(i) Key-Value Databases. Key-value databases [55] consist of a simple data model: data key-value storage. Each key is unique, and the customer can enter the value of the query based on the key. This database structure is simple; modern key-value databases are highly scalable and have query response times that are shorter than those of relational databases. Dynamo [56] is a freely available key-value storage system, and some of Amazon's core services offer an “always-on" experience. To achieve this level of availability, Dynamo sacrifices consistency in certain failure scenarios. It extensively utilizes objectversioning and application-assisted conflict resolution in a way that offers developers a new interface.

Redis [57] is an open-source, support network, memorybased, and optionally persistent key-value pair storage database written in ANSI C. Similar to Memcached, Redis supports storing relatively many value types, including strings, lists, sets, zset (sorted set), and hashes. These data types support push pop, add/remove and intersection, union and difference sets, and richer operations, all of which are 
atomic. Based on this, Redis supports a variety of different sorts. Similar to Memcached, data are cached in memory for efficiency. The difference is that Redis periodically writes updated data to the disk or writes modifications to additional log files and implements master-slave synchronization based on this.

(ii) Column-Oriented Databases. Column-oriented databases [55] store and process data based on columns rather than rows. Columnar storage allows more accurate access to the data being queried, especially in large datasets.

HBase (Hadoop Database) [58] is a high-reliability, highperformance, column-oriented, scalable distributed storage system. Just as Bigtable takes advantage of the distributed data storage provided by Google's file system, HBase provides Bigtable-like capabilities over Hadoop. HBase is a subproject of Apache's Hadoop project and is different from general relational databases, which are suitable databases for unstructured data storage.

(iii) Document Storage. Document storage [55] can support more complex data forms than key-value storage. Because documents do not follow strict patterns, pattern migration is unnecessary. In addition, key-value pairs can still be saved.

MongoDB [59] is a product between a relational database and a nonrelational database. It is the richest and most relational database among nonrelational databases. The data structure that it supports is very loose and is a json-like bson format; thus, one can store more complex data types. MongoDB's most important feature is that it supports a very powerful query language, the syntax is somewhat similar to the object-oriented query language, it can achieve most functions of a relational database single table query, and it supports the indexing of data.

Popular Distributed File Storage systems include HDFS and Colossus. The Hadoop [60] Distributed File System (HDFS) is designed to be suitable for distributed file systems running on commodity hardware. HDFS is the foundation of Hadoop applications' primary data storage, therein distributing files across $64 \mathrm{MB}$ blocks of data and storing them on different nodes in a cluster for parallel computing of MR. The HDFS cluster includes a single NameNode for managing the file system's metadata and a DataNode for storing the actual data. A file is divided into one or more blocks, which are stored in the DataNode. A copy of the block is assigned to a different DataNode to prevent data loss.

Colossus [61] is the successor to the Google file system (GFS). Colossus eliminates the "single point of failure" that plagues the original Google file system. Colossus will also reduce the size of the data block to $1 \mathrm{MB}$ and include multiple primary nodes, which allows Google to store more files on more machines.

5.2. Distributed Parallel Computing Module. Distributed computing is a new method of computation. The so-called distributed computing is whereby two or more software programs can share information with each other; the software can run on the same computer or on multiple computers connected to a network. This method can reduce the overall calculation time and greatly improve the computational efficiency.

The Distributed Parallel Computing module [35] analyzes and processes data from DFS and eventually discovers the information. DPC provides offline computing for a large amount of unstructured data, supports real-time data analysis and querying, and integrates various data mining and machine learning algorithms. DPC supports both real-time analysis and offline analysis.

Real-time analysis [35]: in the context of intensive care, emergency disease detection, or vital sign monitoring, changing data reflect personal health statuses in real time. Therefore, these data need to be promptly processed, and the results of the analysis are expected to be quickly responded to in case of emergencies. Through a memory analysis framework, heart rate, blood pressure, and other related data as well as other important data are recorded to improve the efficiency of the analysis.

Offline analysis [35]: in situations where there are no strong response time requirements (e.g., health assessments, medical recommendations, and health planning), common offline analysis methods in DPC, including machine learning, statistical analysis and recommendation algorithms, can be used to provide individuals with a potential health risk assessment, efficacy analysis, health guidance, etc.

Mainstream distributed computing frameworks include Hadoop, Apache Spark, BOINC, and Apache Storm.

The Hadoop framework [62] transparently provides reliability and data movement for applications. Hadoop implements a programming paradigm called MapReduce: applications are partitioned into many smaller parts, and each part can be run or rerun on any node in the cluster. In addition, Hadoop provides a distributed file system to store data for all compute nodes, bringing very high bandwidths to the entire cluster. MapReduce and the distributed file system design allow the entire framework to automatically handle node failures. It connects applications with thousands of individually calculated computers and petabytes of data.

Apache Spark [63] is an open-source cluster computing framework originally developed by the University of California, Berkeley, AMPLab. MapReduce, in comparison to Hadoop, stores the mediation data to disk after it has finished working. Spark uses in-memory arithmetic to analyze the data in memory when the data have not been written to the hard disk. Spark runs programs in memory at up to $100 \mathrm{x}$ faster than Hadoop MapReduce. Spark can even run up to 10x faster when running programs on the hard disk. Spark allows users to load data into the cluster memory and query them many times, making Spark ideal for machine learning algorithms.

BOINC (Berkeley open network computing platform) [64] is a mainstream distributed computing platform and is a distributed computing system developed by the University of California at Berkeley Computer Science Department. Originally designed for the SETI@home project, the fields currently using BOINC include mathematics, medicine, astronomy, and meteorology. BOINC brings together computers and mobile devices from volunteers around the world to provide computing power to researchers. 


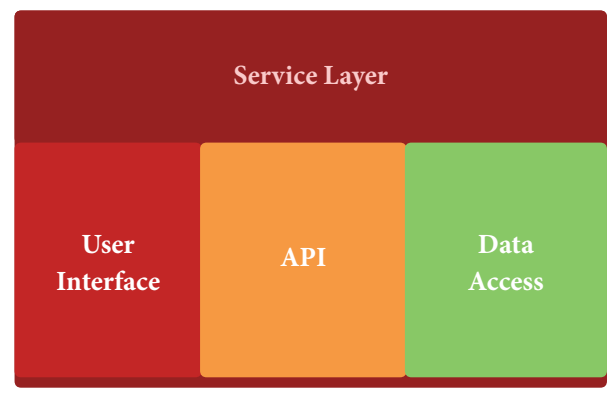

FIGURE 5: The function of service layer.

Apache Storm [65] is a distributed computing framework written mainly in the Clojure programming language. Originally created by Nathan Marz and his team at BackType, the project opened up after being made available on Twitter. It uses user-created "spouts" and "bolts" to define sources and operations to enable the streaming of data in bulk and in a distributed manner. The Storm application is designed to be a topology whose interface creates a transformation "flow". It provides similar functionality as a MapReduce job, and in the event of an exception, the topology theoretically runs indefinitely until it is manually terminated.

\section{Service Layer}

6.1. Main Function of Service Layer. The system is expected to provide a variety of applications and services for different roles (hospitals, patients, wearable device manufacturers, research institutes, pharmaceutical manufacturers, etc.)

As shown Figure 5, the main function of the service layer has the following three points:

(i) User Interface. The user interface provides a unified interface for the user, which makes the interaction and exchange of information between the user and system convenient and provides rich, professional, and personalized medical services.

(ii) API. The API provides a unified application programming interface for developers, which makes programming easy for developers.

(iii) Data Access. Medical data not only come from multiple sources, such as hospitals, research institutes, pharmaceutical companies, and patients, but also have different structures, i.e., being structured, semistructured, or unstructured. Data access provides a unified data access interface for these multisource heterogeneous data.

6.2. Framework of Service Layer. As shown in Figure 6, the service layer framework consists of three parts, namely, the operating platform, the management platform, and the development platform.

The operating platform is the foundation of the service layer, providing the essential resources for running healthcare applications, i.e., hardware, software and data. Hardware can include memory, software can include application software and operating systems, and data can include personal health data, clinical test data, and data on the efficacy of medicine.

The management platform is responsible for managing various applications in the system, including configuration management, deployment management, optimization management, monitoring management, visualization management, and privilege management.

(i) Configuration management is responsible for managing configuration parameters related to the system such as configuration parameter changes.

(ii) Deployment management is responsible for deploying environments and components, which are necessary for system operation.

(iii) Optimization management is responsible for configuring various types of resources within the system efficiently and selecting the final combination that most improves system performance.

(iv) Monitoring management is responsible for monitoring system operations in real time, monitoring user requests, and making judgments on the priority of requests to ensure the stable and normal operation of the system.

(v) Visualization management is responsible for providing multiple modes and ways of displaying data because there may be different targets or target users, and developers need a wide range of graphical tools.

(vi) Privilege management is responsible for assigning authority in a system that provides services for a variety of roles, and different roles have different permissions.

The development platform is responsible for providing a unified API, data access, and a testing platform for developers. Unified API: to reduce the complexity of the system, improve efficiency, and allow developers to more easily program, providing a unified API is necessary.

Data access: ddata are an essential factor in an application. Data access is a process during which developers are allowed to link to the data source through the application to access the data and process the data after returning to the application.

Testing platform: the testing platform is a platform for developers to evaluate the quality of application products and detect weaknesses in the application.

6.3. Data-Oriented Healthcare Services. According to their technical complexity and commercial value, the applications can be divided into the following four groups:

(i) Statistics-based application services only provide basic statistics and report services. The general approach is to first determine a time period, form the statistics of the data within the time period, and finally draw the corresponding report. Personal health status reports are representative applications. For example, it is well known that there is a health app on the Apple iPhone. Whether you are calculating carbohydrates, calories, caffeine, or other important 


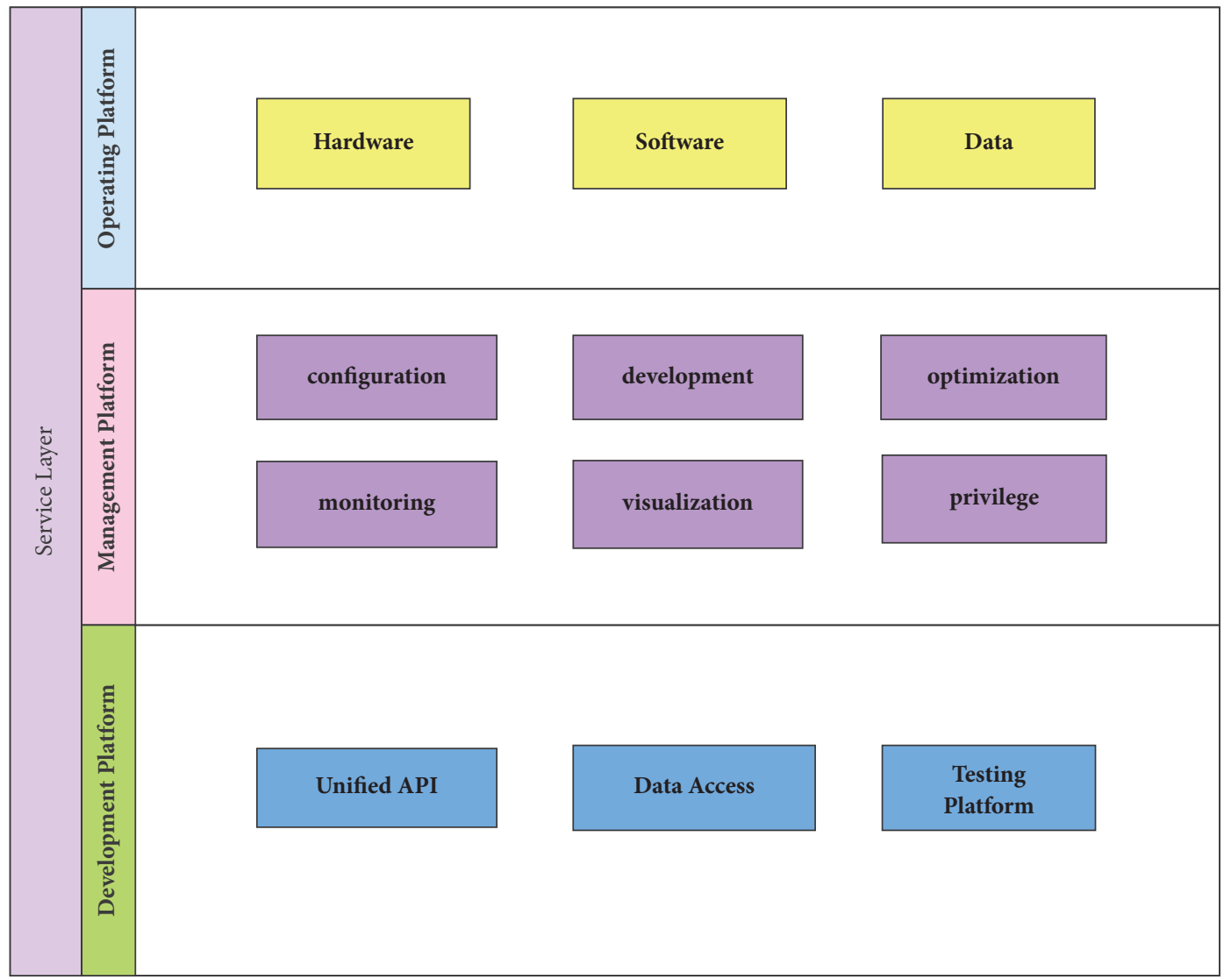

FIGURE 6: The framework of the service layer.

nutritional indicators, health apps can easily manage your goals, check your daily dietary intake, and graphically display them. Therefore, you can keep track of whether your nutritional intake is up to standards and understand the advantages and disadvantages of your diet. In addition, the statistical information posted on social networks reflects the emotional status of individuals and can be used in mental health measures and emotional calculations. For example, De Choudhury M. et al. [66] presented a statistical methodology to identify whether an individual engaged in mental health discourse on social media is likely to transition to that around suicidal ideation in the future. They leveraged a large dataset from a number of mental health and suicide support communities on Reddit to address their research problem. Their method can detect the obvious signs of turning to a suicidal ideation.

(ii) Monitoring-based application services are often used to monitor individual vital signs. Through realtime analysis, one can discover a user's physiological changes in time to avoid sudden illnesses. For example, Luca Catarinucci et al. [46] proposed a novel, IoT-aware, intelligent system for automatically monitoring and tracking patients and personnel and biomedical devices within hospitals and care facilities.
The system can monitor a patient's physiological parameters and environmental conditions in real time and send them to the control center. The control center analyzes the data received and sends an alert message after an exception is detected. Through offline analysis of historical data, recovery procedures can be tracked to support treatment optimization. For example, Giancarlo Fortino et al. [67] proposed a system architecture, Body Cloud, which integrates Body Sensor Network (BSN) services with the cloud computing infrastructure. Body Cloud is an SaaS architecture that supports the storage and management of sensor data streams and offline analysis of stored data using software services hosted in the cloud, thereby allowing physicians to make timely adjustments for treatment plans.

(iii) Knowledge-based application services are the most representative of big data applications. With the support of data mining and machine learning technologies, data dependencies and dependencies can be found. Typical applications include the diagnosis of chronic diseases, genetic disease analysis, treatment evaluation, side effect identification, and public health alerts. For genetic disease analysis, Bravo et al. [68] proposed a novel text mining system called Be Free, which aims to identify the relationships between 
biomedical entities, with particular attention paid to genes and their associated diseases. By exploiting the morphosyntactic information of the text, Be Free is able to identify gene-disease, drug-disease, and drugtarget associations with state-of-the-art performance. They demonstrate the value of the gene-disease associations extracted by Be Free through a number of analyses and integration with other data sources. Be Free succeeds in identifying genes associated with a major cause of morbidity worldwide, depression, which are not present in other public resources. In [69], Lu et al. propose a unified big data processing framework based on the level set evolution method for wound image segmentation, to maximize the advantages of traditional level set models.

(iv) Predictive-based application services have the highest technical complexity and the greatest business value. For example, personal retail spending records reflect lifestyle habits and can predict some potential health risks, especially diet-related illnesses such as obesity and hypertension. In addition, according to individual physical characteristics, personality characteristics and other factors can be used to predict individual preferences and develop medical plans to meet various needs. For example, Yin Zhang et al. [70] developed iDoctor, a new healthcare referral system based on a hybrid matrix factorization approach. iDoctor predicts users' sentiments and preferences by mining user reviews and evaluations of physicians, thereby providing users with specialized, personalized doctor referrals. iDoctor improves the accuracy of healthcare advice significantly by providing a higher forecast rating.

\section{Applications}

7.1. Medical Recommendations. In healthcare, the development of the vulnerability to a disease is often considered permanent, but some patients may change from week to week and even become robust again. However, once established, vulnerability is almost impossible to reverse, and less than $1 \%$ of hospitalized patients were found to have returned during a five-year follow-up period. Hospital readmission, medical costs, institutionalization, and mortality rates will be greatly enhanced. Therefore, we need to promote positive and healthy aging and incitement measures to prevent vulnerability. From a practical point of view, targeting fragility represents a reasonable method. In particular, the use of multivariate interventions to screen, monitor, and manage prefragility-associated precursors, such as subjective or mild cognitive impairment, can be effectively achieved by mobile medical treatments. Corresponding mobile medical equipment [71], considering the timely diagnosis of the patient's physical condition and reflections, can effectively avoid numerous accidents, reducing the patient's number of adverse health outcomes.

Today, data-driven thinking and methods play a key role in the emergence of personalized medicine. Many diseases have preventable risk factors or at least are dangerous.
Clarifying these disease characteristics may help to not only improve personalized healthcare but also reduce the burden of disease. However, the combination of possible risk factors is so complex that it is impossible for an individual physician to analyze it completely (in real time) during patient interactions. Currently, a provider will carefully examine the patient's medical history and perform physical examinations and selective laboratory tests to determine the patient's health condition and future disease risk. These diseases are usually confined to a few diseases, as well as the skills and knowledge of individual providers and the priorities defining individual visits. Thus, taking the next step in personalized healthcare requires the calculation and analysis of big data aggregation and integration frameworks, discovering deep insights into patient similarities and connections, and providing personalized disease risk profiles for each patient's health in a summarized manner[72].

With the increasing use of contemporary mobile messaging data, the mobility of communications technologies and information is also being greatly enhanced. Mobile healthcare, called mobile health or mHealth, has drawn the attention of many practitioners, researchers, and policymakers. Mobile health has the potential to revolutionize healthcare, especially in countries with inadequate medical infrastructure and services in low-income and middleincome low-resource settings [73].

In [70], mixing matrix factorization-based medical recommendations are proposed; advice based on mining the user's evaluations and judgment on the doctor's emotion and preferences developed to provide users with professional, personalized medical treatment is recommended. Specifically, the proposed scheme makes the following contributions to intelligent healthcare services:

(i) A sentiment analysis module, which can calculate a user's emotional state.

(ii) A topic modeling module, which is used to extract the distribution of user preferences and doctor features.

(iii) A hybrid matrix factorization module, which is integrated with two feature distributions extracted by LDA for rating prediction.

7.2. Disease Detection Assisted by Data Analytics. The amount of health-related data has risen sharply in recent years. It is also worth mentioning that the medical insurance reimbursement model is changing, and in today's healthcare environment, meaningful use and performance pay are becoming important new factors. Although profits are not and should not be the main incentive factors, for medical institutions, it is essential to obtain available tools, infrastructure, and technology that can effectively use big data; otherwise, revenues and profits may decrease substantially. Big data includes a variety of features, such as diversity and speed, and it has specific requirements for healthcare. Existing analytical techniques can be applied to a large number of currently unanalyzed health and health data related to patients to better understand the results and then apply them in nursing. Ideally, personal data will inform every doctor and their 
patient in the decision-making process and help determine the most appropriate treatment plan [31].

In recent years, the healthcare system in the United States has been rapidly adopting electronic health records, which will greatly increase the amount of clinical data available electronically. Simultaneously, rapid progress has also been made in clinical analysis techniques to analyze large amounts of data and to gather new insights from the analyses. As a result, we have unprecedented opportunities to reduce the cost of healthcare by using mass data. Here are six key use cases that can reduce costs using big data: high-cost patients, readmissions, shunts, decompensation (when the patient's condition is deteriorating), adverse events, and treatment to optimize conditions affecting multiple organ systems. We discuss the type of opinions that may be obtained from the clinical analysis of the types of data that are required to obtain these insights as well as the infrastructure analysis, algorithms, registries, assessment scores, monitoring equipment, etc. Organizations will need to undertake the necessary analysis and implement improvement measures to improve care and reduce costs [74].

Numerous studies have shown that big data analysis has great potential for improving patient care. However, the use of big data in healthcare is still in its infancy and evidence to date suggests that big data analysis will improve outcomes of care to a minimal extent. However, if big data analysis shows improved quality of care and patient outcomes and can be successfully implemented in practice, big data will see its full potential as a significant component of learning in a healthcare system [75].

7.3. Wearable Healthcare Systems. The 21st century is the information age. In a few short years, with the development of $3 \mathrm{G}$ and $4 \mathrm{G}$, the progress of information technology has had a tremendous impact on all walks of life. The use of smartphones and tablets has changed the fields of communications, commerce, and entertainment. The technology is changing the way healthcare is delivered, including the quality of patient experience and the cost of healthcare. Mobile technology is improving chronic disease management, empowering the elderly and expectant mothers, reminding people to provide services to underserved areas, and improving the health and efficiency of the healthcare system when it is most needed [76].

To better integrate mobile healthcare into our daily life, we adopt wearable devices, wearable sensor systems are likely to produce more than we can currently easily organize, and our ability to explain these dataset is insufficient. To successfully use wearable sensor data to estimate health status and realize improved health management, we must set standards and ontologies between study groups and business systems to share data and promote the integration of these data and health information systems. However, policies and regulations will need to ensure that the details of wearable sensor data are not abused to violate individual privacy or discriminate [77].

To make mobile healthcare easier, more functional, and more comfortable, we consider flexible and scalable sensors that have recently become an active area of research in wearable, implantable, and resorbable systems for mobile health (mHealth) for achieving extensive and unobtrusive health monitoring. Despite this, there is a lack of systematic research comparing the performance of these new sensors to that of conventional sensors. A novel technique that guides the future design of sensors by printing serpentine, flexible, and retractable electrodes that are attached to the skin during the transfer process and using area density (AD) as a key parameter has been developed. These sensors are used to capture an electrophysiological signal, the electrocardiogram (ECG), and are different from when the ECG is obtained with conventional gold and stainless steel metal clips. As a result of this study, flexible sensors designed for larger capture areas yield higher signal-to-noise ratios (SNRs). In particular, ECGs comparable to conventional metal clips (SNR of $25 \mathrm{~dB}$ ) can be achieved with this new flexible sensor design, while the new flexible sensor has a design value of $40 \%$. Thus, this new wearable and flexible electrode design can be used not only for human sensing but also for internal measurements of the gastrointestinal tract [78].

In general, today's wearable health monitoring systems may include various types of microsensors, wearable devices, and even implants. These biosensors are capable of measuring vital physiological parameters such as heart rate, blood pressure, body and skin temperature, oxygen saturation, respiratory rate, and electrocardiogram. Measurements are made by wireless or wired connections to a central node such as a personal digital assistant (PDA) or board; then, they are displayed on a user interface or disseminated to the medical center as aggregated vital signs. In our example, we illustrate the fact that wearable medical systems may include a wide variety of components: sensors, wearable materials, smart textiles, actuators, power supplies, wireless communication modules and links, control and processing units, user interfaces, software, and advanced algorithms for data extraction and decision making [79].

Finally, after a research study, we found Wearable 2.0 to be a better choice for today's mobile healthcare. The Wearable 2.0 front-end system includes a wide range of sensors and serves as a data source for the long-term collection of data that are important to health. Moreover, its front-end system can also function as a user interface. In addition, to achieve a good user experience, medical robots can be presented in the implementation of the front-end system. In particular, mobile robots and human enclosures can provide friendlier and more personalized medical services. For example, when an individual suffers a heart attack and the user loses verbal ability, a medical robot can send a video recording and a picture to a telemedicine center or immediate family member. In addition, walking-capable humanoid games play an important role in emotional interaction when emotion sensing services are required. In this way, the integration of smart garments and humanoid robots helps to improve the interoperability of the system in a variety of complex situations. With the support of mobile cloud systems, big data analysis of healthcare big data during long-term storage and in the cloud can greatly enhance the intelligence and awareness of humanoid robots. Therefore, real-time emotional 
human-computer interaction can be used with human-robotbased support to provide a certain understanding of the user's intentions by the user. Moreover, a robot can also collect environmental data as a mobile receiver. In short, smart garments support high mobility, while robots provide efficient data sensing and health monitoring. Thus, from a number of perspectives, Wearable 2.0 is our best choice for researching mobile healthcare.

\section{Conclusion}

With the development of data analytics and mobile computing, healthcare systems are able to provide more intelligent and convenient applications and services. Moreover, assisted by machine learning, data mining, artificial intelligence, and other advanced techniques, healthcare systems could also play an important role as a guide of healthy lifestyles, as a tool to support decision making, and as a source of innovation in the evolving healthcare ecosystem. This paper presents the intelligent healthcare systems assisted by data analytics and mobile computing, therein consisting of the data collection layer, the data management layer, and the service layer. This paper also introduces some representative applications based on the proposed scheme, which have been proved or demonstrated to be able to provide more intelligent, professional, and personalized healthcare services.

Although this paper presents a comprehensive system design for intelligent healthcare systems assisted by data analytics and mobile computing, more advanced techniques should be included in our future work, such as cognitive computing, deep learning, and affective computing, to further improve the quality of service and user experience.

\section{Conflicts of Interest}

The authors declare that they have no conflicts of interest.

\section{Acknowledgments}

This work was supported by the China National Natural Science Foundation under Grant 61702553 and the Project of Humanities and Social Sciences (17YJCZH252) funded by the China Ministry of Education (MOE).

\section{References}

[1] M. Chen, J. Yang, Y. Hao, S. Mao, and K. Hwang, "A 5G Cognitive System for Healthcare," Big Data and Cognitive Computing, vol. 1, no. 1, p. 2, 2017.

[2] M. M. Hassan, K. Lin, X. Yue, and J. Wan, "A multimedia healthcare data sharing approach through cloud-based body area network," Future Generation Computer Systems, vol. 66, pp. 48-58, 2017.

[3] A. M. Rahmani, T. N. Gia, B. Negash et al., "Exploiting smart eHealth gateways at the edge of healthcare Internet-of-Things: A fog computing approach," Future Generation Computer Systems, vol. 78, pp. 641-658, 2018.

[4] M. Chen, Y. Ma, Y. Li, D. Wu, Y. Zhang, and C.-H. Youn, "Wearable 2.0: Enabling Human-Cloud Integration in Next
Generation Healthcare Systems," IEEE Communications Magazine, vol. 55, no. 1, pp. 54-61, 2017.

[5] P.-L. Wu, W. Kang, A. Al-Nayeem, L. Sha, R. B. Berlin Jr., and J. M. Goldman, "A low complexity coordination architecture for networked supervisory medical systems," in Proceedings of the 4th ACM/IEEE International Conference on Cyber-Physical Systems, ICCPS 2013, pp. 89-98, usa, April 2013.

[6] G.-H. Kim, S. Trimi, and J.-H. Chung, "Big-data applications in the government sector," Communications of the ACM, vol. 57, no. 3, pp. 78-85, 2014.

[7] K. Lee, T. T. H. Wan, and H. Kwon, "The relationship between healthcare information system and cost in hospital," Personal and Ubiquitous Computing, vol. 17, no. 7, pp. 1395-1400, 2013.

[8] M. Chen, "NDNC-BAN: supporting rich media healthcare services via named data networking in cloud-assisted wireless body area networks," Information Sciences, vol. 284, no. 10, pp. 142-156, 2014.

[9] M. Chen, S. Mao, and Y. Liu, "Big data: A survey," Mobile Networks and Applications, vol. 19, no. 2, pp. 171-209, 2014.

[10] H. Zhang, S. Mehotra, D. Liebovitz, C. A. Gunter, and B. Malin, "Mining deviations from patient care pathways via electronic medical record system audits," ACM Transactions on Management Information Systems (TMIS), vol. 4, no. 4, 2013.

[11] R. Mehmood, M. A. Faisal, and S. Altowaijri, "Future networked healthcare systems: A review and case study," Handbook of Research on Redesigning the Future of Internet Architectures, pp. 531-55, 2015.

[12] M. I. Pramanik, R. Y. K. Lau, H. Demirkan, and M. A. K. Azad, "Smart health: Big data enabled health paradigm within smart cities," Expert Systems with Applications, vol. 87, pp. 370-383, 2017.

[13] M. M. Rathore, A. Paul, A. Ahmad, M. Anisetti, and G. Jeon, "Hadoop-Based Intelligent Care System (HICS)," ACM Transactions on Internet Technology (TOIT), vol. 18, no. 1, pp. 1-24, 2017.

[14] S. V. B. Peddi, P. Kuhad, A. Yassine, P. Pouladzadeh, S. Shirmohammadi, and A. A. N. Shirehjini, "An intelligent cloudbased data processing broker for mobile e-health multimedia applications," Future Generation Computer Systems, vol. 66, pp. 71-86, 2017.

[15] R. Nambiar, R. Bhardwaj, A. Sethi, and R. Vargheese, "A look at challenges and opportunities of Big Data analytics in healthcare," in Proceedings of the 2013 IEEE International Conference on Big Data, Big Data 2013, pp. 17-22, usa, October 2013.

[16] J. P. McGlothlin and L. Khan, "Managing evolving code sets and inte- gration of multiple data sources in health care analytics," in Proceedings of the in Proceedings of the 2013 international workshop on Data management analytics for healthcare, p. 14, 2013.

[17] V. Chandola, S. R. Sukumar, and J. Schryver, "Knowledge discovery from massive healthcare claims data," in Proceedings of the 19th ACM SIGKDD International Conference on Knowledge Discovery and Data Mining, KDD 2013, pp. 1312-1320, usa, August 2013.

[18] R. Gravina, P. Alinia, H. Ghasemzadeh, and G. Fortino, "Multisensor fusion in body sensor networks: State-of-the-art and research challenges," Information Fusion, vol. 35, pp. 1339-1351, 2017.

[19] P.-Y. Wu, C.-W. Cheng, C. D. Kaddi, J. Venugopalan, R. Hoffman, and M. D. Wang, "-Omic and Electronic Health Record Big Data Analytics for Precision Medicine," IEEE Transactions on Biomedical Engineering, vol. 64, no. 2, pp. 263-273, 2017. 
[20] C.-H. Lin, L.-C. Huang, S.-C. T. Chou, C.-H. Liu, H.-F. Cheng, and I.-J. Chiang, "Temporal event tracing on big healthcare data analytics," in Proceedings of the 3rd IEEE International Congress on Big Data, BigData Congress 2014, pp. 281-287, usa, July 2014.

[21] M. G. Rabiul Alam, E. J. Cho, E.-N. Huh, and C. S. Hong, "Cloud based mental state monitoring system for suicide risk reconnaissance using wearable bio-sensors," in Proceedings of the 8th International Conference on Ubiquitous Information Management and Communication, ICUIMC 2014, khm, January 2014.

[22] C. He, X. Fan, and Y. Li, "Toward ubiquitous healthcare services with a novel efficient cloud platform," IEEE Transactions on Biomedical Engineering, vol. 60, no. 1, pp. 230-234, 2013.

[23] V. Pejovic and M. Musolesi, "Anticipatory mobile computing: A survey of the state of the art and research challenges," ACM Computing Surveys, vol. 47, no. 3, 2015.

[24] J. Gikas and M. M. Grant, "Mobile computing devices in higher education: Student perspectives on learning with cellphones smartphones \& social media," Internet \& Higher Education, vol. 19, pp. 18-26, 2013.

[25] C. L. Ventola, "Mobile devices and apps for health care professionals: Uses and benefits," Pharmacy Therapeutics, vol. 39, no. 5, pp. 356-64, 2014.

[26] S. Kumar, W. Nilsen, M. Pavel, and M. Srivastava, "Mobile health: revolutionizing healthcare through transdisciplinary research," The Computer Journal, vol. 46, no. 1, Article ID 6357165, pp. 28-35, 2013.

[27] Y.-H. Kao, B. Krishnamachari, M.-R. Ra, and F. Bai, "Hermes: Latency optimal task assignment for resource-constrained mobile computing," in Proceedings of the 34th IEEE Annual Conference on Computer Communications and Networks, IEEE INFOCOM 2015, pp. 1894-1902, hkg, May 2015.

[28] S. Altowaijri, R. Mehmood, and J. Williams, "A quantitative model of grid systems performance in healthcare organisations," in Proceedings of the 2010 International Conference on Intelligent Systems, Modelling and Simulation, pp. 431-436, January 2010.

[29] M. Viceconti, P. Hunter, and R. Hose, "Big Data, Big Knowledge: Big Data for Personalized Healthcare," IEEE Journal of Biomedical and Health Informatics, vol. 19, no. 4, pp. 1209-1215, 2015.

[30] Z. Lv, J. Chirivella, and P. Gagliardo, "Bigdata oriented multimedia mobile health applications," Journal of Medical Systems, vol. 40, no. 5, pp. 1-10, 2016.

[31] W. Raghupathi and V. Raghupathi, "Big data analytics in healthcare: promise and potential," Health Information Science \& Systems, vol. 2, no. 1, p. 3, 2014.

[32] A. Belle, R. Thiagarajan, S. M. R. Soroushmehr, F. Navidi, D. A. Beard, and K. Najarian, "Big data analytics in healthcare," BioMed Research International, vol. 2015, Article ID 370194, 2015.

[33] C.-J. Su and C.-Y. Chiang, "Iaserv: An intelligent home care web services platform in a cloud for aging-in-place," International Journal of Environmental Research and Public Health, vol. 10, no. 11, pp. 6106-6130, 2013.

[34] I. A. T. Hashem, I. Yaqoob, N. B. Anuar, S. Mokhtar, A. Gani, and S. Ullah Khan, "The rise of 'big data' on cloud computing: review and open research issues," Information Systems, vol. 47, pp. 98-115, 2015.

[35] Y. Zhang, M. Qiu, C.-W. Tsai, M. M. Hassan, and A. Alamri, "Health-CPS: Healthcare cyber-physical system assisted by cloud and big data," IEEE Systems Journal, vol. 11, no. 1, pp. 8895, 2017.
[36] L. A. Tawalbeh, R. Mehmood, E. Benkhlifa, and H. Song, "Mobile Cloud Computing Model and Big Data Analysis for Healthcare Applications," IEEE Access, vol. 4, pp. 6171-6180, 2016.

[37] J. Wan, C. Zou, S. Ullah, C.-F. Lai, M. Zhou, and X. Wang, "Cloud-Enabled wireless body area networks for pervasive healthcare," IEEE Network, vol. 27, no. 5, pp. 56-61, 2013.

[38] N. Sultan, "Making use of cloud computing for healthcare provision: opportunities and challenges," International Journal of Information Management, vol. 34, no. 2, pp. 177-184, 2014.

[39] A. Grunerbl, A. Muaremi, V. Osmani et al., "Smart-phone based recognition of states and state changes in bipolar disorder patients," Biomedical \& Health Informatics IEEE Journal, vol. 19, no. 1, pp. 140-148, 2015.

[40] M. M. Rodgers, V. M. Pai, and R. S. Conroy, "Recent advances in wearable sensors for health monitoring," IEEE Sensors Journal, vol. 15, no. 6, pp. 3119-3126, 2015.

[41] M. Chen, Y. Zhang, Y. Li, M. M. Hassan, and A. Alamri, "AIWAC: affective interaction through wearable computing and cloud technology," IEEE Wireless Communications Magazine, vol. 22, no. 1, pp. 20-27, 2015.

[42] Z. Zhang, Z. Pi, and B. Liu, "TROIKA: A general framework for heart rate monitoring using wrist-type photoplethysmographic signals during intensive physical exercise," IEEE Transactions on Biomedical Engineering, vol. 62, no. 2, pp. 522-531, 2015.

[43] A. Pyattaev, K. Johnsson, S. Andreev, and Y. Koucheryavy, "Communication challenges in high-density deployments of wearable wireless devices," IEEE Wireless Communications Magazine, vol. 22, no. 1, pp. 12-18, 2015.

[44] H. Ghasemzadeh, P. Panuccio, S. Trovato, G. Fortino, and R. Jafari, "Power-aware activity monitoring using distributed wearable sensors," IEEE Transactions on Human-Machine Systems, vol. 44, no. 4, pp. 537-544, 2014.

[45] S. Amendola, R. Lodato, S. Manzari, C. Occhiuzzi, and G. Marrocco, "RFID technology for IoT-based personal healthcare in smart spaces," IEEE Internet of Things Journal, vol. 1, no. 2, pp. 144-152, 2014.

[46] L. Catarinucci, D. de Donno, L. Mainetti et al., "An IoT-aware architecture for smart healthcare systems," IEEE Internet of Things Journal, vol. 2, no. 6, pp. 515-526, 2015.

[47] B. Y. Xu, L. D. Xu, H. M. Cai, C. Xie, J. Y. Hu, and F. Bu, "Ubiquitous data accessing method in iot-based information system for emergency medical services," IEEE Transactions on Industrial Informatics, vol. 10, no. 2, pp. 1578-1586, 2014.

[48] G. Yang, L. Xie, M. Mäntysalo et al., "A health-IoT platform based on the integration of intelligent packaging, unobtrusive bio-sensor, and intelligent medicine box," IEEE Transactions on Industrial Informatics, vol. 10, no. 4, pp. 2180-2191, 2014.

[49] S. A. Haque, S. M. Aziz, and M. Rahman, "Review of cyberphysical system in healthcare," International Journal of Distributed Sensor Networks, vol. 2014, Article ID 217415, 2014.

[50] X. Hu, T. H. S. Chu, H. C. B. Chan, and V. C. M. Leung, "Vita: a crowdsensing-oriented mobile cyber-physical system," IEEE Transactions on Emerging Topics in Computing, vol. 1, no. 1, pp. 148-165, 2013.

[51] A. Costanzo, A. Faro, D. Giordano, and C. Pino, "Mobile cyber physical systems for health care: Functions, ambient ontology and e-diagnostics," in Proceedings of the 13th IEEE Annual Consumer Communications and Networking Conference, CCNC 2016, pp. 972-975, usa, January 2016. 
[52] J. Schobel, R. Pryss, M. Schickler, and M. Reichert, “Towards flexible mobile data collection in healthcare," in Proceedings of the 29th IEEE International Symposium on Computer-Based Medical Systems, CBMS 2016, pp. 181-182, irl, June 2016.

[53] P. D. Kaur and I. Chana, "Cloud based intelligent system for delivering health care as a service," Computer Methods and Programs in Biomedicine, vol. 113, no. 1, pp. 346-359, 2014.

[54] A. Gaggioli, G. Pioggia, G. Tartarisco et al., "A mobile data collection platform for mental health research," Personal and Ubiquitous Computing, vol. 17, no. 2, pp. 241-251, 2013.

[55] M. Chen, S. Mao, Y. Zhang, and V. C. Leung, "Related Technologies," in Big Data, SpringerBriefs in Computer Science, pp. 11-18, Springer International Publishing, Cham, 2014.

[56] G. DeCandia, D. Hastorun, M. Jampani et al., "Dynamo: amazon's highly available key-value store," in Proceedings of the 21st ACM Symposium on Operating Systems Principles (SOSP '07), pp. 205-220, ACM, October 2007.

[57] J. Han, E. Haihong, G. Le, and J. Du, "Survey on NoSQL database," in Proceedings of the 6th International Conference on Pervasive Computing and Applications (ICPCA '11), pp.363-366, Port Elizabeth, South Africa, October 2011.

[58] L. George, HBase - The Definitive Guide: Random Access to Your Planet-Size Data, DBLP, 2011.

[59] K. Chodorow and M. Dirolf, MongoDB: The Definitive Guide Powerful and Scalable Data Storage, DBLP, 2010.

[60] D. Borthakur, Hdfs Architecture Guide, 2008.

[61] J. C. Corbett, J. Dean, M. Epstein et al., "Spanner: Googles globally-distributed database," in Proceedings of the Usenix Conference on Op-erating Systems Design and Implementation, pp. 251-264, 2012.

[62] K. Shvachko, H. Kuang, S. Radia, and R. Chansler, "The Hadoop distributed file system," in Proceedings of the IEEE 26th Symposium on Mass Storage Systems and Technologies (MSST '10), 10, 1 pages, Piscataway, NJ, USA, May 2010.

[63] J. G. Shanahan and L. Dai, "Large scale distributed data science using apache spark," in Proceedings of the 21st ACM SIGKDD Conference on Knowledge Discovery and Data Mining, KDD 2015, pp. 2323-2324, aus, August 2015.

[64] D. P. Anderson, "BOINC: a system for public-resource computing and storage," in Proceedings of the 5th IEEE/ACM International Workshop on Grid Computing, pp. 4-10, IEEE, November 2004.

[65] A. G. Shoro and T. R. Soomro, "Big data analysis: Apache spark perspective," Big data analysis: Apache spark perspective, vol. 15, 2015.

[66] M. C. De, E. Kiciman, M. Dredze et al., "Discovering shifts to suicidal ideation from mental health content," in Proceedings of the CHI Conference on Human Factors in Computing Systems, pp. 2098-2110, 2016.

[67] G. Fortino, D. Parisi, V. Pirrone, and G. Di Fatta, "BodyCloud: a SaaS approach for community body sensor networks," Future Generation Computer Systems, vol. 35, pp. 62-79, 2014.

[68] À. Bravo, J. Piñero, N. Queralt-Rosinach, M. Rautschka, and L. I. Furlong, "Extraction of relations between genes and diseases from text and large-scale data analysis: Implications for translational research," BMC Bioinformatics, vol.16, no. 1, article no. 55, 2015.

[69] H. Lu, B. Li, J. Zhu et al., "Wound intensity correction and segmentation with convolutional neural networks," Concurrency and Computation: Practice and Experience, vol. 29, no. 6, p. e3927, 2017.
[70] Y. Zhang, M. Chen, D. Huang, D. Wu, and Y. Li, "IDoctor: personalized and professionalized medical recommendations based on hybrid matrix factorization," Future Generation Computer Systems, vol. 66, pp. 30-35, 2017.

[71] R. O'Caoimh, D. W. Molloy, C. Fitzgerald et al., "Healthcare recommendations from the personalised ict supported service for independent living and active ageing (PERSSILAA) study," in Proceedings of the 3rd International Conference on Information and Communication Technologies for Ageing Well and e-Health, ICT4AWE 2017, pp. 91-103, prt, April 2017.

[72] F. F. Costa, "Big data in biomedicine," Drug Discovery Therapy, vol. 19, no. 4, pp. 433-440, 2014.

[73] A. Chib, M. H. Van Velthoven, and J. Car, "MHealth adoption in low-resource environments: A review of the use of mobile healthcare in developing countries," Journal of Health Communication, vol. 20, no. 1, pp. 4-34, 2015.

[74] D. W. Bates, S. Saria, L. Ohno-Machado, A. Shah, and G. Escobar, "Big data in health care: using analytics to identify and manage high-risk and high-cost patients," Health Affairs, vol. 33, no. 7, pp. 1123-1131, 2014.

[75] J. S. Rumsfeld, K. E. Joynt, and T. M. Maddox, "Big data analytics to improve cardiovascular care: Promise and challenges," Nature Reviews Cardiology, vol. 13, no. 6, pp. 350-359, 2016.

[76] D. West, How Mobile Devices Are Transforming Healthcare How Mobile Devices Are Transforming Healthcare, Washington, Wash, USA, 2012.

[77] S. J. Redmond, N. H. Lovell, G. Z. Yang et al., "What Does Big Data Mean for Wearable Sensor Systems? Contribution of the IMIA Wearable Sensors in Healthcare WG," Yearbook of Medical Informatics, vol. 9, pp. 135-142, 2014.

[78] N. Luo, J. Ding, N. Zhao, B. H. K. Leung, and C. C. Y. Poon, "Mobile health: Design of flexible and stretchable electrophysiological sensors for wearable healthcare systems," in Proceedings of the 11th International Conference on Wearable and Implantable Body Sensor Networks, BSN 2014, pp. 87-91, che, June 2014.

[79] A. Pantelopoulos and N. G. Bourbakis, "A survey on wearable sensor- based systems for health onitoring and prognosis," IEEE Transactions on Systems Man Cybernetics Part C, vol. 40, p. 12, 2009. 


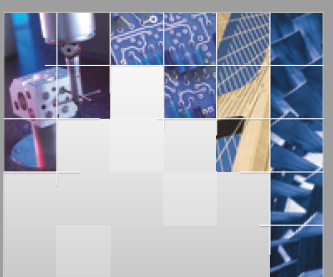

\section{Enfincering}
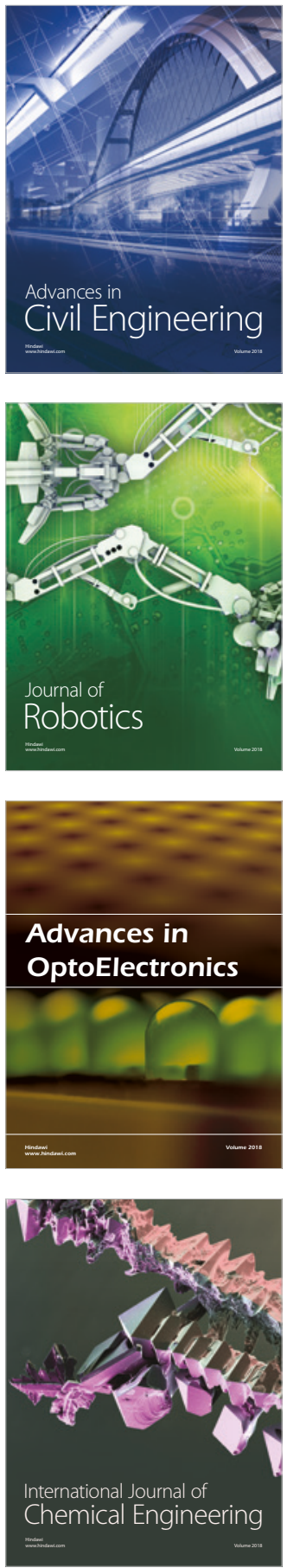

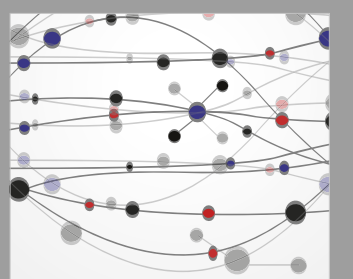

\section{Rotating \\ Machinery}

The Scientific World Journal

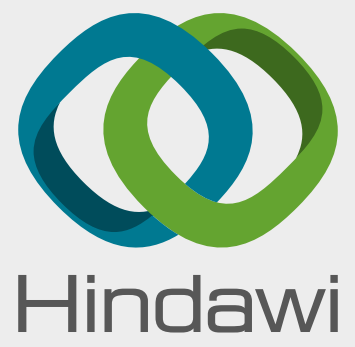

Submit your manuscripts at

www.hindawi.com
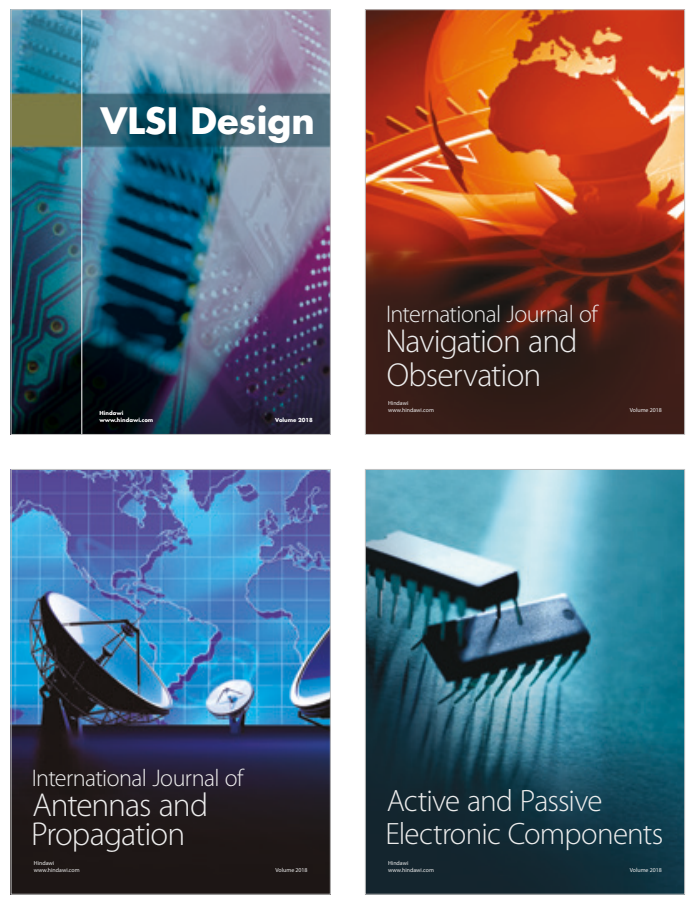
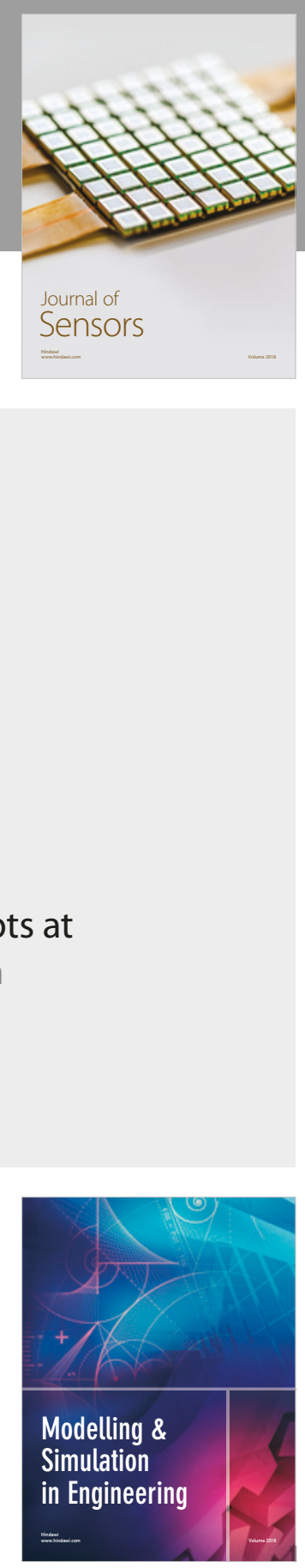

\section{Advances \\ Multimedia}
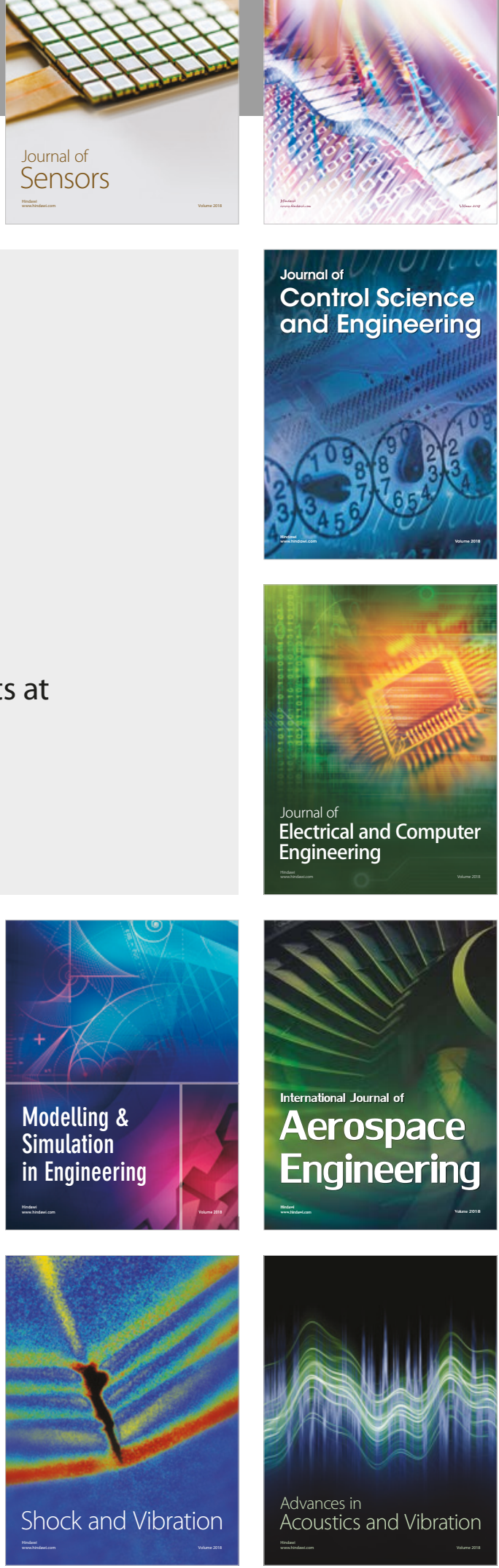